\title{
CUIDADO À SAÚDE DA MULHER NA EXTENSÃO UNIVERSITÁRIA: ABORDAGEM DE UMA EXPERIÊNCIA
}

\author{
Débora Schimitt Porto Fernandes ${ }^{a}$ \\ Geana Silva dos Santos Hübner ${ }^{b}$ \\ Andreia Buffon ${ }^{c}$ \\ Julia Stüker Cezard \\ Luciane Noal Calile
}

\begin{abstract}
Resumo
Atividades de educação em saúde incentivam a prevenção, a detecção precoce de doenças e o desenvolvimento do autocuidado da população feminina, por meio do conhecimento e disponibilidade de exames preventivos. O objetivo deste artigo foi descrever a experiência das autoras no desenvolvimento de uma atividade de extensão interdisciplinar com disponibilização de consultas de enfermagem, que incluíram coleta do citopatológico, exame clínico das mamas e exames laboratoriais junto à população feminina universitária. Tratou-se de estudo transversal, descritivo envolvendo docentes, funcionários técnicos-administrativos (enfermeiros), alunos da Escola de Enfermagem e da Faculdade de Farmácia da Universidade Federal do Rio Grande do Sul. Os resultados mostraram que foram realizadas 56 consultas de enfermagem, que incluíram coleta do citopatológico, exame clínico das mamas e exames laboratoriais. Concluiu-se que o projeto "Saúde da Mulher na Universidade" atingiu o objetivo principal de sensibilizar as mulheres quanto à importância da realização dos exames regulares e a adoção de medidas preventivas contra o desenvolvimento de doenças crônicas.

a Enfermeira. Coordenadora do Laboratório de Práticas de Enfermagem. Escola de Enfermagem. Universidade Federal do Rio Grande do Sul. Porto Alegre, Rio Grande do Sul, Brasil.

b Enfermeira. Mestre em Engenharia de Produção. Coordenadora do Laboratório de Práticas de Enfermagem, Escola de Enfermagem da Universidade Federal do Rio Grande do Sul. Porto Alegre, Rio Grande do Sul, Brasil.

c Farmacêutica-Bioquímica. Mestre e Doutora em Ciências Biológicas. Docente da Faculdade de Farmácia, Universidade Federal do Rio Grande do Sul. Porto Alegre, Rio Grande do Sul, Brasil.

d Graduanda em Enfermagem. Faculdade de Farmácia, Universidade Federal do Rio Grande do Sul. Porto Alegre, Rio Grande do Sul, Brasil.

e Farmacêutica-bioquímica. Mestre em Ciências Farmacêuticas. Doutorado em Medicina: Ciências Médicas. Professora Adjunta do Departamento de Análises, Faculdade de Farmácia, Universidade Federal do Rio Grande do Sul. Porto Alegre, Rio Grande do Sul, Brasil.

Endereço para correspondência: Luciane Noal Calil. Avenida Ipiranga, número 2752, Faculdade de Farmácia, Universidade Federal do Rio Grande do Sul.Rio Grande do Sul, Brasil. CEP: 90610-000. E-mail: luciane1011@gmail.com
\end{abstract}


Revista Baiana de Saúde Pública

Palavras-chave: Saúde da mulher. Enfermagem em saúde comunitária. Teste de Papanicolau. Câncer de colo de útero.

\title{
HEALTH CARE OF WOMEN IN UNIVERSITY EXTENSION: APPROACH OF AN EXPERIENCE
}

\begin{abstract}
Health education activities encourage the prevention, early detection of diseases and the development of self-care by the female population, through knowledge and the availability of preventive tests. The objective of this article was to describe the authors' experience in the development of an interdisciplinary extension activity granting access to nursing consultations, which included collection of the cytopathology, clinical examination of the breasts and laboratory tests with the university female population. This was a cross-sectional, descriptive study involving teachers, technical-administrative staff (nurses), students from the School of Nursing and the Faculty of Pharmacy of the Federal University of Rio Grande do Sul. The results showed that 56 nursing consultations including cytopathologic collection, clinical examination of the breasts, and laboratory tests happened. In conclusion, the project "Women's health at university" achieved the main objective of sensitizing women about the importance of regular examinations and the adoption of preventive measures against the development of chronic diseases.
\end{abstract}

Keywords: Women's health. Community health nursing. Papanicolaou test. Cervical cancer.

\section{CUIDADO DE LA SALUD DE LA MUJER EN LA EXTENSIÓN UNIVERSITARIA: ABORDAJE DE UNA EXPERIENCIA}

\begin{abstract}
Resumen
Las actividades de educación en salud incentivan la prevención, la detección precoz de enfermedades y el desarrollo del autocuidado de la población femenina, a través del conocimiento y disponibilidad de exámenes preventivos. El objetivo de este artículo fue describir la experiencia de las autoras en el desarrollo de una actividad de extensión interdisciplinaria con disponibilidad de consultas de enfermería, que incluyeron la recolección del citopatológico, examen clínico de las mamas y exámenes de laboratorio junto a la población femenina universitaria. Se trata de un estudio transversal, descriptivo que envolviendo a docentes,
\end{abstract}


funcionarias técnico-administrativas (enfermeras), alumnas de la Escuela de Enfermería y de la Facultad de Farmacia de la Universidad Federal de Rio Grande del Sul. Los resultados mostraron que se realizaron 56 consultas de enfermería, incluyendo recolección del citopatológico, examen clínico de las mamas, y exámenes de laboratorio. Se concluyó que el proyecto "Salud de la mujer en la universidad" alcanzó el objetivo principal de sensibilizar a las mujeres en cuanto a la importancia de la realización de los exámenes regulares y la adopción de medidas preventivas contra el desarrollo de enfermedades crónicas.

Palabras clave: Salud de las mujeres. Enfermería en salud comunitaria. Prueba de Papanicolaou. Cáncer de cuello de útero.

\section{INTRODUÇÃO}

Atualmente, na prática, as políticas públicas para a população feminina estão voltadas para a prevenção do câncer de mama e do colo uterino, principais causas de morte. Para o Brasil, dados do Instituto Nacional de Câncer (INCA) em 2016, estimaram 16.340 novos casos de câncer cervical e 57.960 novos casos de câncer de mama. Esses são dados preocupantes, visto que os exames de rastreamento são gratuitos e estão disponíveis pela rede do Sistema Único de Saúde (SUS). Já segundo a Organização Mundial de Saúde (OMS), 15 milhões de novos casos de câncer ocorrerão por ano no mundo a partir de 2020. Todavia, o conhecimento científico existente hoje é suficiente para reduzir esse número consideravelmente ${ }^{1-3}$.

Os principais fatores de risco para o câncer de mama são: idade (envelhecimento), fatores relacionados à reprodução, história familiar, consumo de álcool, excesso de peso, sedentarismo, exposição à radiação ionizante e alta densidade do tecido mamário. No entanto, cerca de $30 \%$ dos casos podem ser evitados se foram tomadas medidas protetoras, prática de atividade física, amamentação, alimentação saudável e manutenção do peso corporal. Esses cuidados não excluem a necessidade dos exames preventivos, quais sejam: mamografia bienal, para mulheres de 50 a 69 anos, exame clínico anual a partir dos 40 anos. Entretanto, para mulheres com história familiar de câncer de mama, é indicada a realização desses exames anualmente, a partir dos 35 anos' $^{1}$.

O tipo histológico mais comum encontrado nos casos de câncer de colo uterino é o carcinoma de células escamosas. Apesar da alta incidência, dentre todos os tipos de câncer, esse é o que possui um dos mais elevados potenciais de prevenção e cura (próximo a $100 \%$ ), desde que descoberto e tratado precocemente, por meio do exame citopatológico 
Revista Baiana de Saúde Pública (papanicloalou). Dados do Ministério da Saúde revelam que cerca de 40\% de todas as mulheres brasileiras nunca tenham realizado esse exame ${ }^{4}$. A literatura refere que o principal fator de risco para o desenvolvimento de células precursoras do câncer (Lesões Intraepiteliais de Alto Grau) e do câncer de colo é a infecção pelo Papiloma vírus humano (HPV). Além disso, outros fatores, como imunidade, genética, comportamento sexual, idade, uso de contraceptivos e baixa condição socioeconômica, também podem estar relacionados ${ }^{5}$. O uso de preservativos e a realização periódica do exame citopatólogico convencional, indicado para mulheres com idade entre 25 e 64 anos, são as principais estrategias dos programas de prevenção e rastreamento ${ }^{2,6}$.

No Brasil, o Programa Nacional de Câncer de Colo de Útero e de Mama "Viva Mulher" foi criado em 1996 e reafirmado como prioridade em 2011, com o lançamento do plano de fortalecimento da rede de prevenção, diagnóstico e tratamento do câncer. Os principais eixos de ação desse programa são: controle do câncer do colo do útero, controle do câncer de mama e ampliação e qualificação da assistência oncológica. Para isso, são necessárias ações de controle, como promoção da saúde, prevenção, detecção precoce, tratamento e cuidado paliativo ${ }^{7}$.

A literatura refere algumas razões que levam a população feminina a não aderirem ao programa, em relação à realização do exame citopatológico. Entre essas são citados medo, timidez, falta de tempo, ausência de sintomas, baixa disponibilidade de vagas nos agendamentos e disponibilização de horários que coincidem com o horário do trabalho. Por isso, a necessidade de orientações que promovam o hábito do autocuidado, assim como de buscar parcerias com instituições públicas, a fim de melhorar o acesso das mulheres, trabalhadoras ou não ${ }^{6}$. Com base nesses dados relevantes, aliados à importância do desenvolvimento de intervenções de educação para a saúde e o aumento do conhecimento das mulheres sobre os fatores de risco, associadas à qualidade de vida, é que um grupo de extensionistas da Universidade Federal do Rio Grande do Sul (UFRGS), composto por docentes, discentes e técnicos-administrativos do curso de graduação em Farmácia e Enfermagem, desenvolveu um projeto de extensão interdisciplinar denominado "Saúde da Mulher na Universidade".

As ações de extensão são contínuas, de caráter educativo, social, cultural, científico e tecnológico, articuladas em função dos interesses da comunidade ${ }^{8}$. É parte integrante da dinâmica pedagógica curricular do processo de formação e de produção do conhecimento, numa proposta dialógica e flexível, possibilitando o pensamento crítico acerca das ações ${ }^{7}$. Estas, quando voltadas para a saúde da mulher, visam o despertar para o autocuidado e o desenvolvimento de habilidades e atitudes pessoais favoráveis à saúde em todas as fases da vida. As equipes de saúde, as políticas públicas e as ações universitárias são responsáveis por 
levar à comunidade as ferramentas necessárias para que possa determinar suas próprias metas de saúde e comportamentos visando à qualidade de vida.

Parte-se do pressuposto que a atuação do enfermeiro na educação para a saúde e na acuidade da coleta do material cérvico-vaginal, assim como a qualidade do diagnóstico realizado pelo profissional bioquímico, seja um meio que proporcionará um diagnóstico preciso, o conhecimento de medidas preventivas e a autonomia no cuidado à saúde.

Assim, dada a relevância do tema e a perspectiva de que a universidade irá agregar e dar suporte à comunidade por meio da relação ensino, pesquisa e extensão, este estudo tem o objetivo de descrever a experiência das autoras no desenvolvimento de uma atividade de extensão interdisciplinar com disponibilização de consultas de enfermagem, que incluíram coleta do citopatológico, exame clínico das mamas e exames laboratoriais junto à população feminina universitária.

\section{MATERIAL E MÉTODOS}

Trata-se de estudo transversal, descritivo, realizado mediante práticas vivenciadas na rotina do Laboratório de Análises Clínicas (LAC) da Faculdade de Farmácia da UFRGS.

A ação de extensão ocorreu no período compreendido entre maio de 2012 e janeiro de 2013, sendo o ano de implantação do projeto. A população alvo foram mulheres com idade acima de 18 anos que estudavam ou trabalhavam na UFRGS (inclusive funcionários que prestavam serviço terceirizado à universidade). A captação das participantes ocorreu por meio de cartazes e e-mails disparados para a comunidade universitária. O agendamento das consultas foi realizado por contato via e-mail ou por indicação. Participaram do projeto, dois docentes da Faculdade de Farmácia e uma da Escola de Enfermagem, duas enfermeiras servidoras da universidade, duas alunas da graduação em farmácia e uma da enfermagem.

Os dados foram obtidos por meio de instrumentos previamente elaborados com base na literatura. Foi empregado um questionário semiestruturado de entrada no estudo, como instrumento de coleta de dados, que caracterizou o perfil da paciente agendada. Esse continha questões de identificação, como idade, estado civil, escolaridade, vínculo com a UFRGS; história familiar e pessoal, hábitos de vida; história ginecológica, conhecimento sobre exame preventivo, autoexame de mamas e história atual. Foram coletadas informações acerca da realização prévia dos exames e suas expectativas, assim como foi elaborado um roteiro, utilizado durante a consulta de enfermagem, cujas etapas compreendiam as seguintes ações: anamnese, exame físico direcionado, intervenções, plano de cuidado, coleta de exames 
Revista Baiana de Saúde Pública bioquímicos e hematológicos, coleta do citopatológico para rastreamento do câncer de colo de útero. Ao final do atendimento, foram dadas orientações sobre a coleta de sangue, como tempo de jejum e horário de atendimento. Após a coleta, as amostras eram enviadas para preparo e análise pela equipe.

Os resultados, logo depois da liberação, foram avaliados juntamente com a análise do questionário e o roteiro da consulta, para elaboração do plano de cuidados e encaminhamentos aos serviços de saúde, se necessário. Estabelecia-se contato com as pacientes, para agendamento da consulta, entrega dos resultados e orientações. Além disso, nas reconsultas, era realizada uma pesquisa de opinião, utilizando como referência a escala de Lickert de cinco pontos, que avaliava as seguintes questões: agendamento, qualidade do atendimento, área física, entrevista, coleta de exames, orientações, reconsulta e tempo para entrega dos resultados ${ }^{9}$.

A entrevista para aplicação do questionário e a coleta dos exames foram realizadas uma vez por semana. Eram agendados no máximo seis pacientes/dia. Na entrevista inicial, solicitava-se a autorização das participantes quanto à utilização dos dados, por meio da leitura e assinatura do Termo de Consentimento Livre e Esclarecido (TCLE). Todos os cuidados éticos que regem pesquisas com seres humanos foram tomados, sendo esta registrada no Comitê de Pesquisa da Universidade Federal do Rio Grande do Sul, sob n. 25.572/2012. Não foram citados dados oriundos da avaliação e do resultado dos exames das pacientes, pois deverão ser objeto de publicações posteriores.

O estudo baseou-se na Resolução n. 466/12 do Conselho Nacional de Saúde (CNS), o qual incorpora os referenciais básicos da bioética, bem como os princípios éticos de autonomia, não maleficência, beneficência e justiça ${ }^{10}$.

\section{RESULTADOS E DISCUSSÃO}

Participaram do projeto 56 mulheres. Os dados de perfil etário (Tabela1) indicam que a maioria encontra-se na faixa etária de 18 a 35 anos (67,86\%) e em menor percentual a faixa etária de 46 a 65 anos (14,29\%). Esse achado provavelmente ocorreu em função de que a maioria das participantes era estudante da universidade, em idade adulta jovem. Esse fato também foi observado numa pesquisa em que foram avaliadas 1.501 coletas de exame de Papanicolau numa rede pública municipal ${ }^{11}$. A Tabela 1 apresenta ainda a faixa etária das pacientes, estratificada dos 18 aos 65 anos. A faixa etária predominante nas coletas foi de 20 a 34 anos, representando um total de $43,2 \%$. Este resultado sugere, assim como apresentado na literatura, que o exame preventivo é realizado de forma oportuna nas consultas ginecológicas e obstétricas ${ }^{11}$. 
Tabela 1 - Perfil etário das pacientes atendidas. Porto Alegre, Rio Grande do Sul, Brasil - 2013

\begin{tabular}{l|cc}
\hline Faixa etária & $\mathbf{n}$ & $\mathbf{\%}$ \\
\hline $18-25$ anos & 13 & 23,21 \\
$26-35$ anos & 25 & 44,64 \\
$36-45$ anos & 10 & 17,86 \\
$46-55$ anos & 6 & 10,71 \\
$56-65$ anos & 2 & 3,57 \\
\hline
\end{tabular}

Fonte: Elaboração própria.

Conforme apresentado na Tabela 2, com relação ao estado civil, 30 mulheres eram solteiras (53,57\%), sendo 39,29\% casadas ou em união estável. Esse fato corrobora resultado de estudo que salienta a maior preocupação das mulheres solteiras com o diagnóstico preventivo, em função de não possuírem relacionamento estável ${ }^{12}$. Segundo pesquisa, a sociedade confere às mulheres casadas, em relação às solteiras, a falsa ideia de que são possuidoras de certo grau de imunidade às doenças sexualmente transmissíveis (DSTs) ${ }^{13}$. Este resultado assemelha-se aos de estudo no qual a maioria das mulheres que participaram da pesquisa eram solteiras ${ }^{14}$.

Tabela 2 - Estado civil, grau de escolaridade e vínculo com a universidade. Porto Alegre, Rio Grande do Sul, Brasil - 2013

\begin{tabular}{lcc}
\hline Variáveis & $\mathbf{n}$ & $\mathbf{\%}$ \\
\hline Estado civil & & 53,57 \\
Solteira & 30 & 17,86 \\
Casada & 10 & 21,43 \\
União estável & 12 & 5,36 \\
Divorciada & 3 & 1,79 \\
Viúva & 1 & - \\
Escolaridade & & 1,79 \\
Sem instrução & - & 7,14 \\
Fundamental incompleto & 1 & 10,71 \\
Fundamental completo & 4 & 14,29 \\
Médio incompleto & 6 & 44,64 \\
Médio completo & 8 & 21,43 \\
Universitário incompleto & 25 & \\
Universitário completo & 12 & 8,90 \\
Vínculo com a URFGS & & 60,70 \\
Servidor & 5 & - \\
Estudante & 34 & 30,30 \\
Professor & - & \\
Terceirizado & 11 & \\
\hline
\end{tabular}

Fonte: Elaboração própria.

Nota: Sinal convencional utilizado:

- Dado numérico igual a zero não resultante de arredondamento. 
Revista Baiana de Saúde Pública
Formação universitária incompleta foi apresentada por 44,64\% das mulheres e 21,43\% apresentavam o curso universitário completo; não se teve analfabetas no estudo. Esses resultados eram esperados, já que o público-alvo era proveniente de comunidade universitária, a qual, devido ao nível mais elevado de escolaridade, preocupa-se mais com a saúde e com o autocuidado. Quanto ao vínculo com a UFRGS, 60,7\% eram estudantes e 30,3\% eram funcionárias terceirizadas. Contou-se com a participação de poucos servidores, apenas 8,9\%, provavelmente porque a universidade oferece convênio com plano de saúde para eles.

A Tabela 3 apresenta dados quanto às questões clínico-ginecológicas. Observa-se que a maioria havia feito o último citopatológico num período menor ou igual a um ano $(51,79 \%)$ e $19,64 \%$ já não fazia o exame há três anos ou mais. Somente 5,36\% estavam realizando o exame pela primeira vez. A OMS preconiza a realização de exame citológico do colo uterino a cada três anos, após dois exames anuais consecutivos negativos para neoplasia, para mulheres de 25 a 59 anos ou que já tenha iniciado a vida sexual ${ }^{1-2}$.

Tabela 3 - Questões clínico-ginecológicas das mulheres atendidas. Porto Alegre,

Rio Grande do Sul, Brasil - 2013

\begin{tabular}{lcc}
\hline Variáveis & $\mathbf{n}$ & $\%$ \\
\hline Data do último exame Citopatológico & 3 & 5,36 \\
$\quad$ o Exame & 29 & 51,79 \\
Menos ou igual a 1 ano & 13 & 23,21 \\
2 anos & 11 & 19,64 \\
$\quad$ Mais ou igual a 3 anos & & \\
Uso de Contraceptivo & 20 & 35,71 \\
Nao faz & 25 & 44,64 \\
Pílula & 2 & 3,57 \\
Dispositivo intrauterino & 8 & 14,29 \\
Outros & & 37,50 \\
Uso de Camisinha & 21 & 44,64 \\
Sim & 25 & 17,86 \\
Não & 10 & \\
Às vezes & & \\
\hline
\end{tabular}

Fonte: Elaboração própria.

Ao ser analisado o uso da camisinha nas relações sexuais, encontrou-se que $44,64 \%$ das participantes não utilizava o método. A camisinha masculina é o método de prevenção de gravidez e de Doenças Sexualmente Transmissíveis (DST) mais conhecido e mais usado entre os adolescentes, e os principais motivos alegados para a sua não utilização de modo consistente foram: não gostar de usá-las, confiar no parceiro, perda da libido e imprevisibilidade 
das relações sexuais. Além disso, autores observaram que a assimetria de gênero nas relações entre os adolescentes é percebida na negociação do condom, quando as meninas não sugerem o uso do preservativo com medo de perder o rapaz, provocando uma ruptura na relação ${ }^{19}$.

Das mulheres estudadas, 35,71\% não utilizavam nenhum método contraceptivo. Essa mesma porcentagem foi encontrada para as mulheres que utilizavam pílula anticoncepcional, o que permite inferir-se que, ao utilizarem a pílula, elas não faziam uso da camisinha. Estudo ${ }^{20}$ considera que é alto o percentual de jovens que, ao utilizarem pílulas anticoncepcionais, abrem mão do uso de preservativos, o que corrobora os achados desta pesquisa. Assim, faz-se menção à preocupação existente com relação às práticas preventivas adotadas pelas mulheres, uma vez que, em meio à disseminação das DSTs, ocorre paralelamente uma disseminação da pílula, facilitando o abandono do preservativo, adotado como método anticoncepcional ${ }^{21}$.

A medida preventiva, por excelência, é de ordem comportamental e diz respeito, diretamente, ao uso de preservativo. Cabe, portanto, uma intensificação das estratégias de promoção da saúde sexual junto a essa população.

\section{CONSIDERAÇÕES FINAIS}

O trabalho da inclusão social, reforçando o papel da educação para incentivar o autocuidado com base em projetos que proporcionem o acesso aos exames preventivos e de mama, deve ser enfatizado. Foi com esse propósito que o projeto relatado neste estudo foi realizado.

Após diversas solicitações de servidores, acadêmicos e terceirizados, o projeto de extensão "Saúde da Mulher na Universidade" foi desenvolvido para essa população. Verificou-se que a maioria das ações de extensão era voltada para a comunidade externa, isto é, raras eram as iniciativas voltadas para a promoção da saúde da comunidade universitária.

Foi um projeto pioneiro, promovido pela Escola de Enfermagem e pela Faculdade de Farmácia, ambas da UFRGS, que permitiu não apenas a disponibilização de exames, mas também incentivou os primeiros passos dos alunos de graduação na compreensão do cuidado à saúde da mulher, coleta e análise citopatológica. Reforça-se a importância da parceria do analista clínico no diagnóstico das alterações e do enfermeiro na coleta e orientação quanto ao autocuidado e encaminhamentos, visto que a conquista da interdisciplinaridade entre ensino, pesquisa e extensão representa um dos maiores desafios das universidades brasileiras. Por isso, a discussão sobre esse tema assume importância.

Concluiu-se que o projeto "Saúde da Mulher na Universidade" atingiu o objetivo principal de sensibilizar as mulheres quanto à importância da realização dos exames regulares e 
Revista Baiana de Saúde Pública a adoção de medidas preventivas contra o desenvolvimento de doenças crônicas. Vale destacar que exames preventivos possibilitam o diagnóstico precoce do câncer, tanto de mama quanto de colo de útero, em um estágio inicial da doença, quando o tratamento é potencialmente curativo. Identificaram-se algumas limitações, como dificuldade no contato com as pacientes no agendamento da reconsulta e entrega de exames, no qual seriam realizadas as orientações, bem como informadas medidas preventivas e de incentivo ao autocuidado. $\mathrm{Na}$ entevista realizada, identificaram-se alguns fatores que dificultaram o acesso das mulheres aos resultados dos exames, como: horário de trabalho, dificuldade de comunicação por telefone, esquecimento, atraso na liberação do resultado do exame, entre outros. Evidenciou-se também, em algumas situações, a impossibilidade de encaminhar as pacientes a serviços de saúde de referência, pois, no contato telefônico, verificou-se a não existência de profissionais, nos Postos de Saúde, para dar seguimento ao tratamento e manutenção da saúde.

Pôde-se constatar que o objetivo de proporcionar exames preventivos de fácil acesso à população da universidade foi alcançado com sucesso. Por isso, entende-se que o projeto realizado, com base nos resultados obtidos, foi de suma importância para a melhor compreensão do quadro de saúde das mulheres da UFRGS. Além disso, para os bolsistas envolvidos no projeto, significou melhor qualificação do aprendizado acadêmico.

\section{COLABORADORES}

1. Concepção do projeto, análise e interpretação dos dados: Débora Schimitt Porto Fernandes, Luciane Noal Calil e Julia Stüker Cezar.

2. Redação do artigo e revisão crítica relevante do conteúdo intelectual: Débora Schimitt Porto Fernandes, Andreia Buffon, Julia Stüker Cezar e Luciane Noal Calil.

3. Revisão e/ou aprovação final da versão a ser publicada: Débora Schimitt Porto Fernandes, Geana Silva dos Santos Hübner, Julia Stüker Cezar, Luciane Noal Calil.

4. Ser responsável por todos os aspectos do trabalho na garantia da exatidão e integridade de qualquer parte da obra: Luciane Noal Calil.

\section{REFERÊNCIAS}

1. Brasil. Ministério da Saúde. Instituto Nacional de Câncer. Estimativa 2014: Síntese de resultados e comentários. Rio de Janeiro; 2014 [citado 2014 out 21]. Disponível em: http://www.inca.gov.br/estimativa/2014/sintese-deresultados-comentarios.asp

2. Brasil. Ministério da Saúde. Instituto Nacional de Câncer. Diretrizes brasileiras para o rastreamento do câncer do colo do útero. Rio de Janeiro; 2011 [citado 
2014 ago 13]. Disponível em: http://www1.inca.gov.br/inca/Arquivos/Titulos/ [Nomenclatura_colo_do_utero.pdf]

3. Greenwood AS, Machado MFAS, Sampaio NMV. Motivos que levam mulheres a não retornarem para receber o resultado de exame Papanicolau. Rev Latino-am Enfermagem. 2006;14(4):503-9.

4. Dell'Agnollo CM, Brischiliari SCR, Saldan G, Gravena AAF, Lopes TCR, Demitto MO, et al. Avaliação dos exames citológicos de Papanicolau em usuárias do Sistema Único de Saúde. Rev baiana saúde pública. 2014;38(4):854-64.

5. Albuquerque KM, Frias PG, Andrade CLT, Aquino EML, Menezes G, Szarcwald CL. Cobertura do teste de Papanicolaou e fatores associados à nãorealização: um olhar sobre o Programa de Prevenção do Câncer do Colo do Útero em Pernambuco, Brasil. Cad Saúde Pública. 2009;25(2):301-9.

6. Domingos ACP, Murata LMH, Pelloso SM, Schirmer J, Carvalho MDB. Câncer do colo útero: comportamento preventivo de autocuidado à saúde. Ciênc cuid saúde. 2007;6(2):397-403.

7. Silva MS, Vasconcelos S. Extensão universitária e formação profissional: avaliação da experiência das ciências biológicas na Universidade Federal de Pernambuco. Estudos em avaliação educacional. 2006 [citado 2014 out 21];17(33):119-36. Disponível em: http://www.fcc.org.br/pesquisa/ publicacoes/eae/arquivos/1280/1280.pdf

8. Casarin MR, Piccoli JE. Educação em saúde para prevenção do câncer de colo do útero em mulheres do município de Santo Ângelo/RS. Ciênc saúde coletiva. 2011;16(9):3925-32.

9. Alexandre JWC, Andrade DF, Vasconcelos AP, Araujo MAS, Batista MJ. Análise do número de categorias da escala de Likert aplicada à gestão pela qualidade total através da teoria da resposta ao item. In: 23 Encontro Nacional de Engenheiros de Produção (ENEGEP); 2003 out 21-24. Ouro Preto, MG, Brasil [citado 2014 out 21]. Disponível em: http://www.abepro.org.br/biblioteca/ enegep2003_tr0201_0741.pdf

10. Brasil. Ministério de Saúde. Conselho Nacional de Saúde. Resolução n. 466, de 12 de dezembro de 2012. Aprova as diretrizes e normas regulamentadoras de pesquisas envolvendo seres humanos. Brasília; 2012 [citado 2016 mar 3]. Disponível em: http://bvsms.saude.gov.br/bvs/saudelegis/cns/2013/ res0466_12_12_2012.html

11. Soares MBO, Silva SR. Análise de um programa municipal de prevenção do câncer cérvico-uterino. Rev bras enferm. 2010;63(2):177-82. 
Revista Baiana de Saúde Pública
12. Melo SCCS, Prates L, Carvalho MDB, Marcon SS, Pelloso SM. Alterações citopatológicas e fatores de risco para a ocorrência do câncer de colo uterino. Rev Gaúcha Enferm. 2009;30(4):602-8.

13. Guedes GT, Pordeus AMJ, Diógenes MAR. Análise epidemiológica do cancer de colo de útero em serviço de atendimento terciário no Ceará - Brasil. Rev Bras Promoç Saúde. 2005;18(4):205-10.

14. Ferreira ASS, Arantes Júnior JC, Chaoubah A, Louzada CF, Amorim AG, Vasconcelos YA. Aspectos clínico-epidemiológicos das pacientes portadoras de alterações colpocitologicas atendidas no Hospital Universitário da UFJF. HU Revista. 2011;37(4):421-9.

15. Amorim VMSL, Barros MBA, César CLG, Carandina L, Golbaum M. Fatores associados à não realização do exame de Papanicolau: um estudo de base populacional no Município de Campinas, São Paulo, Brasil. Cad Saúde Pública. 2006;22(11):2329-38.

16. Oliveira IR, Inagaki ADM, Daltro AST, Gonçalves LLC, Santos LV. Práticas preventivas e fatores de risco para câncer cervico uterino entre docentes universitárias. REME rev min enferm. 2009;13(2):238-43.

17. Martins LBM, Costa-Paiva LHS, Osis MJD, Souza MH, Pinto-Neto AM, Tadini V. Fatores associados ao uso de preservativo masculino e ao conhecimento sobre DST/AIDS em adolescentes de escolas públicas e privadas do Município de São Paulo, Brasil. Cad Saúde Pública. 2006;22(2):315-23.

18. Sampaio J, Santos RC, Callou JLL, Souza BBC. Ele não quer com camisinha e eu quero me prevenir: exposição de adolescentes do sexo feminino às DST/Aids no semi-árido. Saúde Soc. 2011;20(1):171-81.

19. Viegas-Pereira APF. Aids? Tô fora: um estudo com adolescentes em duas escolas de Belo Horizonte sobre os fatores que determinam o uso do preservativo na era da AIDS [tese]. Minais Gerais (MG): Universidade Federal de Minas Gerais; 2000 [citado 2014 out 20]. Disponível em: http://www. abep.nepo.unicamp.br/docs/anais/pdf/2000/Todos/Posteres/AIDS\%20Tô\%20 fora\%20-\%20Um\%20Estudo\%20com\%20Adolescentes....pdf

20. Oliveira DC, Pontes APM, Gomes AMT, Ribeiro MCM. Conhecimentos e práticas de adolescentes acerca das DST/HIV/AIDS em duas escolas públicas municipais do Rio de Janeiro. Esc Anna Nery. 2009;13(4):833-41.

21. Chinazzo IR, Câmara SG, Frantz DG. Comportamento sexual de risco em jovens: aspectos cognitivos e emocionais. Psico-USF. 2014;19(1):1-12.

Recebido: 27.10.2015. Aprovado: 4.3.2016. Publicado: 8.11.2017. 